\title{
Bimodal Chromoendoscopy with Confocal Laser Endomicroscopy for the Detection of Early Esophageal Squamous Cell Neoplasms
}

\author{
Piyapan Prueksapanich ${ }^{1}$, Thanawat Luangsukrerk ${ }^{2}$, Rapat Pittayanon ${ }^{1}$, Anapat Sanpavat ${ }^{3}$ and Rungsun Rerknimitr ${ }^{1}$ \\ ${ }^{1}$ Division of Gastroenterology, Department of Medicine, Faculty of Medicine, ${ }^{2}$ Division of General Internal Medicine, Department of Medicine, \\ Faculty of Medicine, ${ }^{3}$ Department of Pathology, Faculty of Medicine, Chulalongkorn University and King Chulalongkorn Memorial Hospital, \\ Bangkok, Thailand
}

Background/Aims: This study aimed to evaluate the diagnostic accuracy of dual-focus narrow-band imaging (dNBI) and Lugol'schromoendoscopy (LCE) combined with probe-based confocal laser endomicroscopy (pCLE) to screen for esophageal squamous cell neoplasms (ESCNs) in patients with a history of head and neck cancer.

Methods: From March to August 2016, dNBI was performed. Next, LCE was performed, followed by pCLE and biopsy. Histology has historically been the gold standard to diagnose ESCN. The sensitivity, specificity, positive predictive value (PPV), negative predictive value (NPV), and accuracy of dNBI and LCE adjunct with pCLE were determined.

Results: Twenty-four patients were included. Ten ESCNs were found in 8 patients (33\%). Forty percent of high-graded intraepithelial neoplasias and all low-grade intraepithelial neoplasias were overlooked by dNBI. The sensitivity, specificity, PPV, NPV, and accuracy of dNBI vs. LCE combined with pCLE were $50 \%$ vs. $80 \%, 62 \%$ vs. $67 \%, 36 \%$ vs. $44 \%, 75 \%$ vs. $91 \%$, and $83 \%$ vs. $70 \%$, respectively.

Conclusions: The use of dNBI to detect ESCN was suboptimal. LCE with pCLE following dNBI had additional value for detecting esophageal dysplasia not detected by dNBI. The use of pCLE to detect dNBI-missed lesions yielded a high NPV, while pCLE-guided biopsy could reduce the number of unnecessary biopsies. Clin Endosc 2019;52:144-151

Key Words: Narrow-band imaging; Lugol's chromoendoscopy; Probe-based confocal laser endomicroscopy; Early esophageal squamous cell neoplasm

\section{INTRODUCTION}

Esophageal cancer is the sixth most common cancer worldwide. ${ }^{1}$ Patients with esophageal squamous cell carcinoma (SCC) have a very low survival rate, mainly due to late detection. Therefore, early detection is important to improve survival rates. ${ }^{2,3}$ Early esophageal squamous cell neoplasms (ESCNs) can be categorized as low-grade intraepithelial neo-

Received: May 30, 2018 Revised: August 9, 2018

Accepted: August 19, 2018

Correspondence: Rungsun Rerknimitr

Division of Gastroenterology, Department of Medicine, Faculty of Medicine, King Chulalongkorn Memorial Hospital, 1873 Rama IV Road, 2nd fl Bhumisirimungkalanusorn Building, Pathum Wan, Bangkok 10330, Thailand

Tel: +66-2-256-4265, Fax: +66-2-256-4265, E-mail: ercp@live.com ORCID: https://orcid.org/0000-0001-6866-6886

cc This is an Open Access article distributed under the terms of the Creative Commons Attribution Non-Commercial License (http://creativecommons.org/ licenses/by-nc/3.0) which permits unrestricted non-commercial use, distribution, and reproduction in any medium, provided the original work is properly cited. plasia (LGIN), high-grade intraepithelial neoplasia (HGIN), and superficial esophageal SCC. For cases of early ESCN, endoscopic treatment can be curative. ${ }^{4}$ Patients with previously diagnosed head and neck SCC are at the highest risk of developing ESCNs; ${ }^{5}$ therefore, the American Society of Gastrointestinal Endoscopy (ASGE) recommends endoscopic surveillance for ESCN in such cases. ${ }^{5}$ However, the ASGE has not mentioned how such surveillance should be performed or at what interval.

Lugol's chromoendoscopy (LCE) is the standard method for detecting ESCNs, which usually appear as Lugol's voiding lesions (LVLs). ${ }^{6}$ Despite its high sensitivity, LCE is not specific only to ESCN since other lesions including inflammatory lesions can mimic LVLs. Thus, unnecessary biopsies are unavoidable, which in turn could increase the cost of ESCN surveillance. In addition, Lugol's solution may irritate the aerodigestive tract. Probe-based confocal laser endomicroscopy (pCLE), which provides a real-time in vivo histology image 
of epithelial cells and the intrapapillary capillary loops (IPCLs) of the esophageal mucosa, is now commercially available. Hypothetically, pCLE may be useful for the further characterization of LVLs as shown in our recent study.

Dual-focus narrow-band imaging (dNBI), which uses selective wavelengths of visible light to enhance the visibility of mucosal lesions and the IPCL, is an image-enhanced endoscopy technique that can be used to screen for ESCNs. ${ }^{8}$ The advantages of dNBI over LCE include its ability to both detect and characterize suspicious mucosal lesions and avoid the irritation caused by Lugol's solution. Some studies showed that dNBI provided similar sensitivity to but higher specificity than LCE. ${ }^{9,10}$ However, those results could be implied for SCC and HGIN but not LGIN. Hence, the overall utilities of LCE versus dNBI for ESCN detection including LGIN have never been compared.

Therefore, this study aimed to evaluate the diagnostic performance of dNBI followed by a combination of LCE and pCLE in dNBI-missed lesions for ESCN surveillance in patients with a previous history of head and neck SCC.

\section{MATERIALS AND METHODS}

\section{Patients}

This study was performed at King Chulalongkorn Memorial Hospital, Bangkok, Thailand, between March and August 2016. Patients with a previous history of histologically confirmed head and neck SCC between January 1, 2013 and December 31, 2015 were enrolled. The inclusion criteria were age $>18$ years and no dysphagia symptoms. The exclusion criteria were the ongoing treatment plan of the original cancer, alteration of esophageal anatomy (history of surgery, stricture, or obstruction), esophageal varices, a known history of esophageal cancer, intractable bleeding diathesis, gravidity, hyperthyroidism, estimated glomerular filtration rate $<30 \mathrm{~mL} /$ $\mathrm{min}$, and a history of hypersensitivity to iodine or fluorescein. Patients with nasopharyngeal SCC were also excluded due to its poor correlation with a second primary ESCN. ${ }^{11}$ Informed consent was provided by all patients. The study protocol was approved by the institutional review board (COA No. 791/2015) and registered with the Thai Clinical Trials Registry (TCTR 20180223004).

\section{Study design}

This was a single-center cross-sectional diagnostic test study of the diagnostic accuracy of dNBI followed by a combination of LCE and PCLE for a second primary ESCN diagnosis in patients with a previous history of head and neck SCC. After standard white-light upper endoscopy (WLE), esoph- agoscopy with dNBI was carefully performed. Any lesions detected by dNBI were biopsied. Next, LCE was performed in the same endoscopic session to detect the dNBI-missed lesions. The LVLs were characterized with pCLE in real-time mode and then biopsied. The histopathological results of the lesions detected by $\mathrm{dNBI}$ and the LVLs were considered the gold standard. The sensitivity, specificity, positive predictive value (PPV), negative predictive value (NPV), and accuracy of $\mathrm{dNBI}$ and LCE combined with PCLE for ESCN detection were calculated.

\section{Instrument and criteria for the diagnosis of ESCN}

All endoscopies were performed using a dual-focus EVIS EXERA III GIF-HQ190 gastrointestinal videoscope (Olympus, Tokyo, Japan). The abnormal dNBI findings suspicious of ESCN were interpreted according to Inoue's classification (Fig. 1). ${ }^{12} \mathrm{~A}$ lesion containing a regular IPCL was considered normal, while a lesion containing an irregular, dilated, and tortuous IPCL was considered neoplastic.

After a careful dNBI examination, LCE was performed after spraying of the esophagus with $0.5 \%$ Lugol's solution. An LVL $>5 \mathrm{~mm}$ was considered a potential neoplasm, ${ }^{13}$ and pCLE was used to further characterize it using a Gastroflex Cellvizio (Mauna Kea Technology, Paris, France) with a 1000× magnified power. The pCLE images were interpreted in real time based on cellular and vascular criteria (Fig. 2). The cellular criteria included a homogenous and regular architecture and clearly visible cell border for normal squamous epithelium and an inhomogeneous and irregular architecture and no clearly visible cell border for neoplastic lesions. The capillary criteria included a regular and non-neoplastic capillary appearance without fluorescein leakage for normal squamous epithelium and an inconsistent caliber, twisting, enlarged, and elongated appearance with some fluorescein leakage for neoplastic lesions. ${ }^{14}$

\section{Procedure}

Intravenous meperidine and midazolam plus an oral anesthetic spray were used to achieve moderate sedation levels to obtain optimal pCLE images. First, WLE was performed. When a noticeable mucosal abnormality such as an elevated or depressed lesion ${ }^{8}$ was seen, lesion size, location, and images were recorded. Next, an dNBI examination of an esophageal mucosa was carefully performed. If a brownish area with abnormal IPCLs was found, lesion size, location, and images were recorded. The first endoscopist (PP) who performed the dNBI had previously performed more than $100 \mathrm{dNBI}$ examinations in many parts of the gastrointestinal tract prior to this study. Every lesion suspected to be an ESCN on dNBI was biopsied for histological confirmation. 

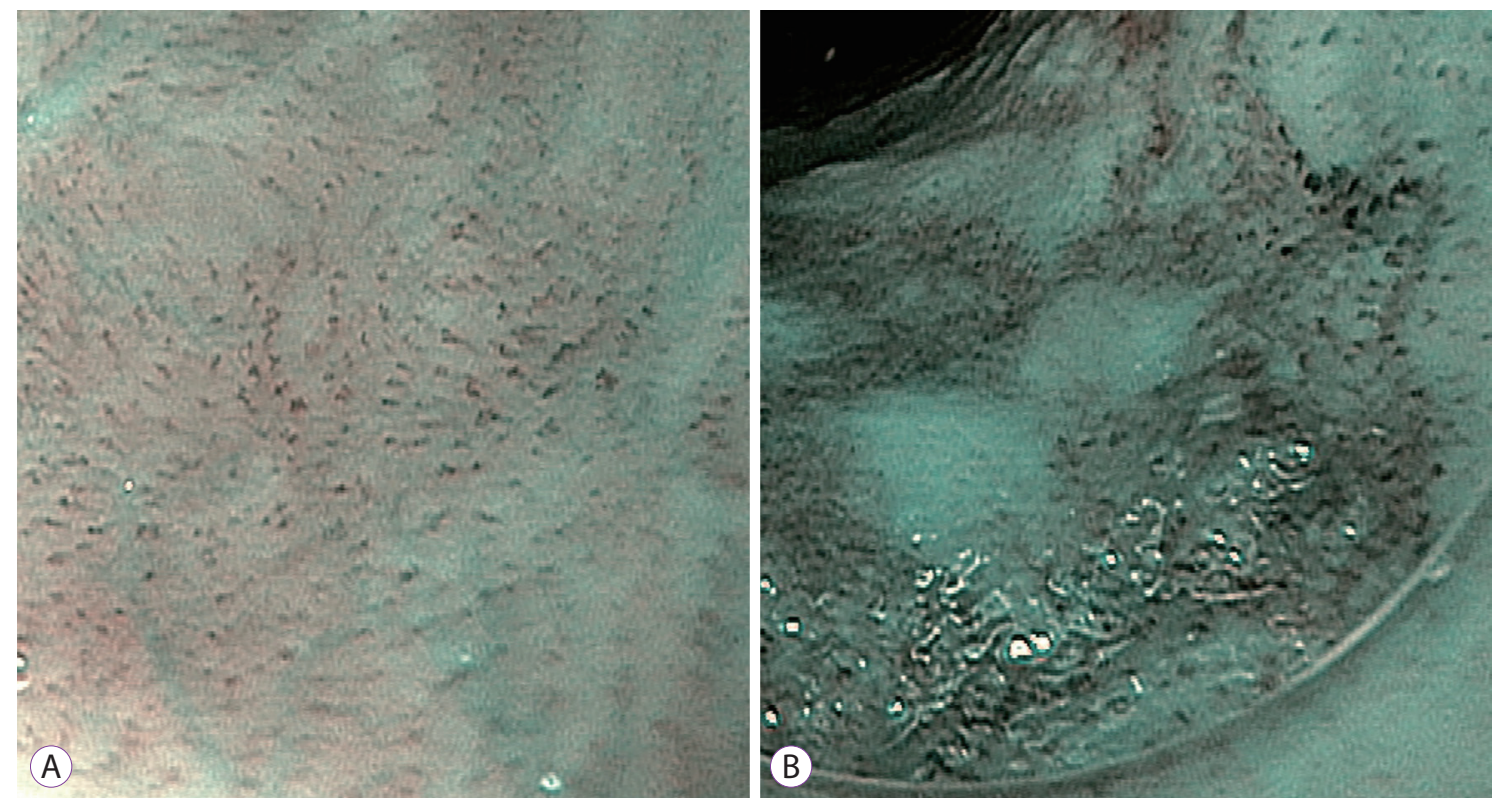

Fig. 1. Near focus view of dual-focus narrow-band imaging (dNBI) of normal esophageal mucosa showing a normal intrapapillary capillary loop (IPCL) pattern (A). The near focus view of dNBI of high-grade intraepithelial neoplasia shows a well-demarcated brownish area with dilated, tortuous, and various shapes of IPCL (B).
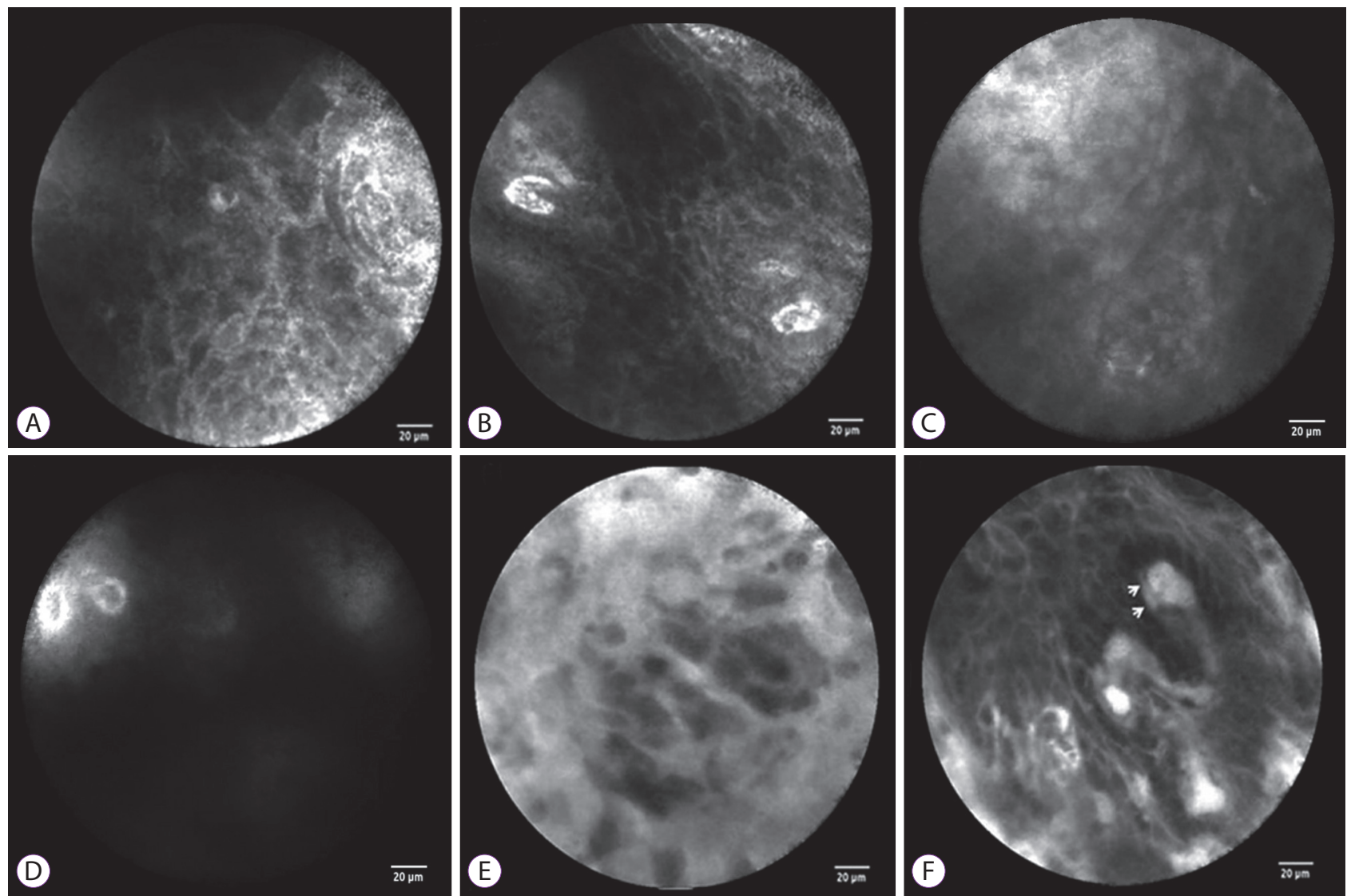

Fig. 2. The probe-based confocal laser endomicroscopy (pCLE) findings of normal esophageal mucosa and esophageal squamous cell neoplasms. For normal esophageal mucosa, pCLE shows a homogenous, regular architecture, clearly visible cell borders (A), and regular capillaries without fluorescein leakage (B). For lowgrade intraepithelial neoplasia, pCLE shows a slightly inhomogeneous, irregular architecture, clearly visible cell borders (C), and regular capillaries without fluorescein leakage (D). For high-grade intraepithelial neoplasia, pCLE shows an inhomogeneous and irregular cellular architecture without clearly visible cell borders (E) and irregular, twisted, dilated, elongated capillaries $(F)$ with fluorescein leakage $(F$, arrows). 
Next, the LCE study with a $0.5 \%$ Lugol's solution spray was performed in the same endoscopic session to detected $\mathrm{dN}$ BI-missed lesions. LVLs $>5 \mathrm{~mm}$ were further examined using pCLE for characterization. Fluorescein was injected intravenously a few seconds prior to the pCLE examination. The second endoscopist (RP) who performed pCLE had performed $>100$ pCLE examinations in many parts of gastrointestinal tract including the esophagus prior to this study. All LVLs and their characteristics from the pCLE results were recorded, and the real-time diagnosis was made according to the pCLE criteria (Fig. 2). Finally, all LVLs with positive or negative pCLE results were biopsied for histological confirmation. The role of pCLE in combination with LCE was to further characterize the LVLs into the neoplastic or non-neoplastic group. However, the performance of LCE combined with PCLE in the dNBI-missed lesions had not been established previously, so the histological result was needed to determine pCLE performance. All tissue biopsies were sent to an expert gastrointestinal pathologist (AS) who was blinded to the endoscopic results.

\section{Sample size and statistical analysis}

Earlier studies reported that dNBI and LCE combined with pCLE had comparable sensitivity. ${ }^{6,10,15}$ To eliminate unnecessary biopsies, we chose to compare the specificity between dNBI and LCE combined with pCLE. In our previous study, ${ }^{7}$ the specificity of LCE combined with pCLE was $92 \%$. The required study population size was calculated by a one-sided binomial test with a $95 \%$ confidence interval. Thirty-two esophageal lesions were required for this study with an alpha probability of 0.05 and desired precision of 0.1 . The baseline characteristics are shown as means and standard deviations for continuous variables and as percentages with frequencies for categorical variables. The sensitivity, specificity, PPV, NPV, and accuracy of dNBI and a combination of LCE with pCLE were calculated using the histology findings as the gold standard. SPSS software version 23.0 for Windows (IBM Corp., Chicago, IL, USA) was used for performing statistical analyses.

\section{RESULTS}

All 587 patients who were previously diagnosed with head and neck SCC were invited to enroll in this study (Fig. 3). Unfortunately, 563 patients were excluded (359 could not be contacted; 107 had died; 10 had an esophageal obstruction, stricture, or surgery; 74 were still undergoing radiation therapy; and 13 refused to participate). Eventually, 24 asymptomatic patients (96\% men; mean age, 63 years) who were previously diagnosed with head and neck SCC were enrolled. The demographic data of all patients are shown in Table 1. Most of our patients were ex-smokers (75\%) with a mean interval of 27 months since the head and neck.

Thirty-four lesions were detected in 18 patients. Of them, 10 lesions (Table 2) in 8 patients (33\%) were histologically diagnosed as ESCN (2 invasive cancers, 5 HGIN, and 3 LGIN). WLE detected only one invasive cancer that appeared as a surface nodularity on the esophageal mucosa (Fig. 4), while dNBI detected a total of 14 lesions suspected to be ESCN in 9 patients. The histological result of dNBI-detected lesions showed 2 invasive cancers, one of which was also detected by WLE and 3 if which were detected by HGIN. The other 9 lesions were benign (esophagitis and squamous papilloma). The sensitivity, specificity, PPV, NPV, and accuracy of dNBI were $50 \%, 62 \%, 36 \%, 75 \%$, and $83 \%$, respectively.

After the dNBI examination, LCE showed 20 LVLs that were not visible on the prior dNBI. Next, pCLE was used to characterize those LVLs and interpreted 9 of 20 lesions as ESCN. The histological result of these LVLs (the dNBI-missed lesions) showed 2 HGIN and 3 LGIN. Thus, dNBI could not detect $40 \%$ of HGIN and dNBI could not detect any LGIN. The sensitivity, specificity, PPV, NPV, and accuracy of LCE combined with pCLE in lesions not detected by dNBI were $80 \%, 67 \%, 44 \%, 91 \%$, and $70 \%$, respectively.

For the ESCN treatment, 2 patients with an invasive cancer were treated by radiotherapy. Endoscopic follow-up showed a complete response after radiotherapy. Endoscopic mucosal resection (EMR) was the treatment of choice in 3 patients with HGIN. The other 2 patients with HGIN chose early scheduled

Table 1. The Baseline Characteristics of the Enrolled Patients

\begin{tabular}{lc}
\hline Gender & \\
Male, $n(\%)$ & $13(96)$ \\
Female, $n(\%)$ & $63(46-82)$ \\
Age, yr (range) & \\
Index Head and Neck SCC & $12(50)$ \\
Glottis, $n(\%)$ & $8(33)$ \\
Oral cavity, $n(\%)$ & $3(13)$ \\
Oropharynx, $n(\%)$ & $1(4)$ \\
Hypopharynx, $n(\%)$ & $27(8-114)$ \\
Mean interval after a primary diagnosis, mo & \\
(range) & \\
Smoking status & $2(8)$ \\
Non-smoker, $n(\%)$ & $4(17)$ \\
Current smoker, $n$ (\%) & $18(75)$ \\
Ex-smoker, $n(\%)$ & \\
\hline
\end{tabular}

SCC, squamous cell carcinoma. 


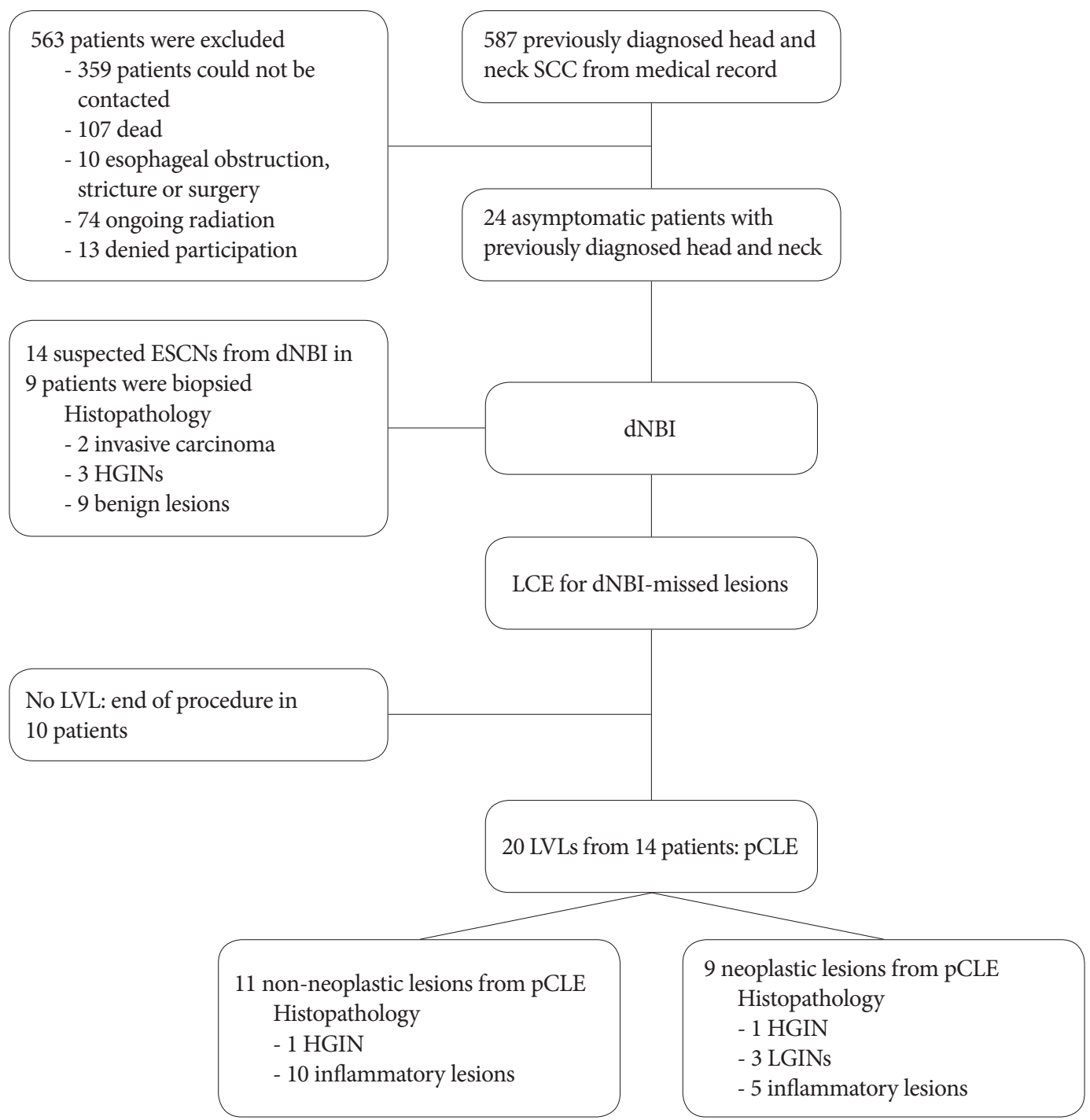

Fig. 3. Flow chart of the studied patients. dNBI, dual-focus narrow-band imaging; ESCN, esophageal squamous cell neoplasm; HGIN, high-grade intraepithelial neoplasia; LCE, Lugol's chromoendoscopy; LGIN, low-grade intraepithelial neoplasia; LVL, Lugol's voiding lesion; pCLE, probe-based confocal laser endomicroscopy; SCC, squamous cell carcinoma.

surveillance. And finally, all patients with LGIN were appointed for endoscopic surveillance every 6-12 months.

\section{DISCUSSION}

Esophageal SCC is the most common second primary tumor in head and neck SCC patients with a 22 -fold higher risk compared with the general population. ${ }^{16}$ The early detection of ESCN in asymptomatic patients on surveillance endoscopy probably improves survival in those patients. ${ }^{11}$ In a previous study, the incidence of second primary ESCN was reportedly up to $44 \%$ in head and neck SCC patients. ${ }^{17}$ Our study result that showed a $33 \%$ prevalence of a second primary ESCN is similar to that of the earlier study. The mean interval after the diagnosis of head and neck SCC was 27 months, which was similar to the series reported by Schwartz et al. (range, 13-42 months).$^{18}$ Due to the very high prevalence, we recommend endoscopic surveillance for ESCN in all patients with head and neck SCC.

This study confirmed the low sensitivity (10\%) of WLE to detect early ESCN in asymptomatic patients with head and neck SCC. ${ }^{15}$ In Japanese studies, NBI showed high sensitivity and specificity for HGIN and SCC diagnosis (sensitivity of $88.3 \%$ and specificity of $75.2 \%$ for non-magnified NBI; sensitivity of $90.9 \%$ and specificity of $95.4 \%$ for magnified NBI). ${ }^{9,10}$ Compared to our results, the sensitivity and specificity for HGIN and invasive cancer surveillance were $71.4 \%$ and $66.7 \%$, respectively. There are some possible reasons for the suboptimal performance of NBI in our study. First, the Japanese stud- 
Table 2. The Characteristics of 8 Patients with Esophageal Squamous Cell Neoplasm and the Detection Methods

\begin{tabular}{|c|c|c|c|c|c|c|c|c|}
\hline Case No. & Sex & Age & $\begin{array}{l}\text { Index head and } \\
\text { neck SCC }\end{array}$ & $\begin{array}{l}\text { Segment of } \\
\text { esophagus }\end{array}$ & Histology & WLE & dNBI & LCE with pCLE \\
\hline 4 & $\mathrm{M}$ & 68 & Oropharynx & Middle & HGIN & Missed & Missed & $\begin{array}{c}\text { LVL+ } \\
\text { Misclassified as non-neoplastic } \\
\text { by pCLE }\end{array}$ \\
\hline \multirow[t]{2}{*}{8} & M & 55 & Glottis & Middle & HGIN & Missed & Missed & $\begin{array}{l}\text { LVL+ } \\
\text { Classified as neoplastic by pCLE }\end{array}$ \\
\hline & & & & Middle & LGIN & Missed & Missed & $\begin{array}{c}\text { LVL+ } \\
\text { Classified as neoplastic by pCLE }\end{array}$ \\
\hline \multirow[t]{2}{*}{11} & $\mathrm{~F}$ & 52 & Glottis & Middle & Invasive cancer & Detected & Detected & LVL- \\
\hline & & & & Middle & HGIN & Missed & Detected & LVL- \\
\hline 12 & M & 71 & Oral cavity & Middle & Invasive & Missed & Detected & LVL- \\
\hline 13 & M & 62 & Glottis & Middle & HGIN & Missed & Detected & LVL- \\
\hline 14 & M & 62 & Glottis & Middle & HGIN & Missed & Detected & LVL- \\
\hline 18 & M & 68 & Glottis & Middle & LGIN & Missed & Missed & $\begin{array}{l}\text { LVL+ } \\
\text { Classified as neoplastic by pCLE }\end{array}$ \\
\hline 19 & M & 62 & Hypopharynx & Middle & LGIN & Missed & Missed & $\begin{array}{c}\text { LVL+ } \\
\text { Classified as neoplastic by pCLE }\end{array}$ \\
\hline
\end{tabular}

dNBI, dual-focus narrow-band imaging; HGIN, high-grade intraepithelial neoplasia; LCE, Lugol's chromoendoscopy; LGIN, low-grade intraepithelial neoplasia; LVL+, presence of Lugol's voiding lesion; LVL-, absence of Lugol's voiding lesion; pCLE, probe-based confocal laser endomicroscopy; SCC, squamous cell carcinoma; WLE, white-light upper endoscopy.

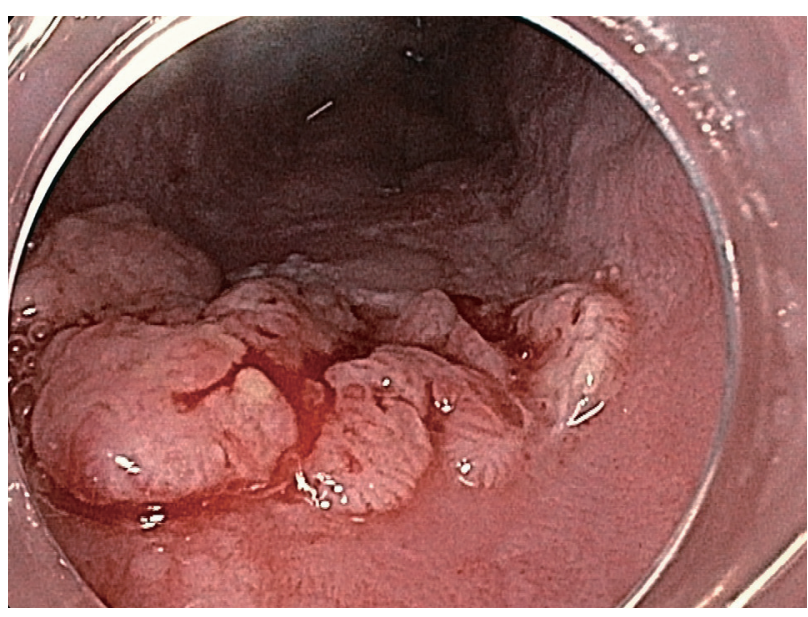

Fig. 4. Squamous cell cancer detected by white-light endoscopy showing a nodular surface with dilated neoplastic vessels.

ies used a different NBI technique and equipment than ours (the EVIS LUCERA NBI system versus the EVIS EXCERA NBI, respectively). Second, magnified NBI might provide an add-on benefit due to its higher sensitivity and specificity than dNBI. Goda et al. ${ }^{19}$ recently showed a higher image resolution with magnified NBI than with dNBI. However, their diagnostic accuracies were similar.

The limitation of NBI is its poor sensitivity to detect LGIN. ${ }^{10,11}$ As described by Inoue et al., ${ }^{12}$ we could not discrim- inate LGIN from an atrophic or inflamed esophageal mucosa because they share similar IPCL patterns. LGIN has been considered a precancerous lesion that possesses a risk of progressing to esophageal SCC with a rate of up to $26.7 \%$ during a 3.5-year follow-up period. ${ }^{20}$ LGIN could be managed by endoscopic resection or scheduled endoscopic surveillance within a short period of time. ${ }^{21}$ However, good evidence of and established guidelines for squamous LGIN management are lacking. Our data emphasized the additional benefit of LCE to detect LGIN not detected by dNBI. In this study, all three LGIN were missed by dNBI but later detected and confirmed using a combination of LCE and pCLE.

LCE, the primary mode for ESCN surveillance, boasts the highest sensitivity for detecting ESCN. ${ }^{6}$ However, the specificity of LCE as the primary mode for ESCN diagnosis is substantially low, ${ }^{6}$ which may result in a high number of unnecessary biopsies. Our study demonstrated the better sensitivity and specificity of LCE ( $80 \%$ and $67 \%$, respectively) as the secondary mode after dNBI than those reported when used as the primary mode. ${ }^{22}$ Of note, pCLE was also used for the further characterization for LVLs in our study; otherwise, our reported specificity of LCE could have been lower. The NPV of LCE combined with PCLE in this study was $91 \%$, which is in line with the results of our earlier trial (92\%) that used LCE with pCLE as the primary mode for ESCN diagnosis. These data imply that when pCLE showed a benign pattern in the 
LVLs, the biopsy used for histological confirmation could possibly be omitted.

When early ESCN was diagnosed, mucosal tumors at the Tis and Tla stage can be treated by EMR without the need for surgery. ${ }^{3}$ In our study, all patients with ESCN were promptly treated. In 2 patients with invasive cancer, esophagectomy could not be performed due to the presence of mediastinal fibrosis from previous head and neck SCC radiotherapy in 1 patient and comorbid alcoholic cirrhosis with portal hypertension in the other. These two patients were treated with radiotherapy. After the radiotherapy sessions were completed, endoscopic follow-up showed complete response in those tumors. EMR was performed in 3 patients with HGIN. The other 2 patients with HGIN refused treatment with EMR and were scheduled for serial surveillance. Finally, all patients with LGIN chose to undergo endoscopic surveillance every 6-12 months.

Our study had two main limitations. First, we did not directly compare the performance of $\mathrm{dNBI}$ to that of LCE combined with pCLE by dividing our patients in two groups because we expected only a handful of enrollees. Therefore, we chose the tandem examination designed by starting with dNBI and then using LCE. In our earlier study, we experienced that even after heavy water irrigation, the residual Lugol's stain could impair the future reading by $\mathrm{dNBI}^{7}$ hence, the crossover tandem approach was not selected. Due to the study's tandem design, this study could not directly compare the diagnostic powers of the modalities; rather, it reported the additional role of LCE followed by dNBI and pCLE for detecting esophageal dysplasia missed by dNBI, especially LGIN. Second, the cost of pCLE is currently very high compared to the cost of biopsy in some countries, including Thailand. However, in the setting in which the cost of biopsy is higher (North America, for example), and multiple lesions require biopsy, real-time in vivo histology by pCLE appears more attractive. Thus, a study comparing the cost-effectiveness of optical biopsy by pCLE versus histological biopsy is required.

In summary, the performance of $\mathrm{dNBI}$ for ESCN surveillance in patients with head and neck SCC was suboptimal because a considerable number of HGIN and all of LGIN were overlooked. The LCE with pCLE following dNBI had additional value for detecting cases of esophageal dysplasia missed by dNBI. Because of its high NPV, adding pCLE to further characterize LVLs is recommended to avoid unnecessary biopsies.

\section{Author Contributions}

Conceptualization: Piyapan Prueksapanich, Rapat Pittayanon, Rungsun Rerknimitr

Data curation: Thanawat Luangsukrerk
Formal analysis: TL

Investigation: PP, TL, RP, Anapat Sanpavat

Methodology: PP, TL, RP, RR

Writing-original draft: PP, TL

Writing-review\&editing: RP, RR

Conflicts of Interest

The authors have no financial conflicts of interest.

\section{Acknowledgments}

This work was funded by the Ratchadapiseksompotch Fund, Faculty of Medicine, Chulalongkorn University (grant number RA59/021) and The Gastroenterological Association of Thailand.

\section{REFERENCES}

1. Jemal A, Bray F, Center MM, Ferlay J, Ward E, Forman D. Global cancer statistics. CA Cancer J Clin 2011;61:69-90.

2. Wang GQ, Jiao GG, Chang FB, et al. Long-term results of operation for 420 patients with early squamous cell esophageal carcinoma discovered by screening. Ann Thorac Surg 2004;77:1740-1744.

3. Rustgi AK, El-Serag HB. Esophageal carcinoma. N Engl J Med 2014;371:2499-2509.

4. Katada C, Muto M, Momma K, et al. Clinical outcome after endoscopic mucosal resection for esophageal squamous cell carcinoma invading the muscularis mucosae--a multicenter retrospective cohort study. Endoscopy 2007;39:779-783.

5. Hirota WK, Zuckerman MJ, Adler DG, et al. ASGE guideline: the role of endoscopy in the surveillance of premalignant conditions of the upper GI tract. Gastrointest Endosc 2006;63:570-580.

6. Freitag CP, Barros SG, Kruel CD, et al. Esophageal dysplasias are detected by endoscopy with Lugol in patients at risk for squamous cell carcinoma in southern Brazil. Dis Esophagus 1999;12:191-195.

7. Prueksapanich P, Pittayanon R, Rerknimitr R, Wisedopas N, Kullavanijaya P. Value of probe-based confocal laser endomicroscopy (pCLE) and dual focus narrow-band imaging (dNBI) in diagnosing early squamous cell neoplasms in esophageal Lugol's voiding lesions. Endosc Int Open 2015;3:E281-E288.

8. Lee CT, Chang CY, Lee YC, et al. Narrow-band imaging with magnifying endoscopy for the screening of esophageal cancer in patients with primary head and neck cancers. Endoscopy 2010;42:613-619.

9. Takenaka R, Kawahara Y, Okada H, et al. Narrow-band imaging provides reliable screening for esophageal malignancy in patients with head and neck cancers. Am J Gastroenterol 2009;104:2942-2948.

10. Nagami $Y$, Tominaga $K$, Machida $H$, et al. Usefulness of non-magnifying narrow-band imaging in screening of early esophageal squamous cell carcinoma: a prospective comparative study using propensity score matching. Am J Gastroenterol 2014;109:845-854.

11. Chen MC, Feng IJ, Lu CH, et al. The incidence and risk of second primary cancers in patients with nasopharyngeal carcinoma: a population-based study in Taiwan over a 25-year period (1979-2003). Ann Oncol 2008;19:1180-1186.

12. Inoue $\mathrm{H}$, Kaga $\mathrm{M}$, Ikeda $\mathrm{H}$, et al. Magnification endoscopy in esophageal squamous cell carcinoma: a review of the intrapapillary capillary loop classification. Ann Gastroenterol 2015;28:41-48.

13. Hashimoto CL, Iriya K, Baba ER, et al. Lugol's dye spray chromoendoscopy establishes early diagnosis of esophageal cancer in patients with primary head and neck cancer. Am J Gastroenterol 2005;100:275-282.

14. Liu H, Li YQ, Yu T, et al. Confocal laser endomicroscopy for superficial esophageal squamous cell carcinoma. Endoscopy 2009;41:99-106.

15. Lee YC, Wang CP, Chen CC, et al. Transnasal endoscopy with nar- 
row-band imaging and Lugol staining to screen patients with head and neck cancer whose condition limits oral intubation with standard endoscope (with video). Gastrointest Endosc 2009;69:408-417.

16. Day GL, Blot WJ. Second primary tumors in patients with oral cancer. Cancer 1992;70:14-19.

17. Priante AV, Castilho EC, Kowalski LP. Second primary tumors in patients with head and neck cancer. Curr Oncol Rep 2011;13:132-137.

18. Schwartz LH, Ozsahin M, Zhang GN, et al. Synchronous and metachronous head and neck carcinomas. Cancer 1994;74:1933-1938.

19. Goda K, Dobashi A, Yoshimura N, et al. Dual-focus versus conventional magnification endoscopy for the diagnosis of superficial squamous neoplasms in the pharynx and esophagus: a randomized trial. Endoscopy
2016;48:321-329.

20. Dawsey SM, Lewin KJ, Wang GQ, et al. Squamous esophageal histology and subsequent risk of squamous cell carcinoma of the esophagus. A prospective follow-up study from Linxian, China. Cancer 1994;74:16861692.

21. Su YY, Chen WC, Chuang HC, et al. Effect of routine esophageal screening in patients with head and neck cancer. JAMA Otolaryngol Head Neck Surg 2013;139:350-354.

22. Li J, Xu R, Liu M, et al. Lugol chromoendoscopy detects esophageal dysplasia with low levels of sensitivity in a high-risk region of China. Clin Gastroenterol Hepatol 2018;16:1585-1592. 